\title{
Forward modelling of inherent optical properties from flow cytometry estimates of particle size and refractive index
}

\author{
JACOPO AgAGLIATE, ${ }^{1}$ INA LeFERING, ${ }^{1}$ DAVID McKeE ${ }^{1, *}$ \\ ${ }^{1}$ Department of Physics, University of Strathclyde, 107 Rottenrow, Glasgow, G4 ONG, Scotland \\ *Corresponding author: david.mckee@strath.ac.uk
}

Received XX Month XXXX; revised XX Month, XXXX; accepted XX Month XXXX; posted XX Month XXXX (Doc. ID XXXXX); published XX Month XXXX

\begin{abstract}
A Mie-based forward modelling procedure was developed to reconstruct bulk inherent optical properties (IOPs) from particle size distributions (PSDs) and real refractive index distributions (PRIDs) obtained using a previously developed flow cytometric (FC) method [1]. Given the available PSDs, extrapolations for the particle fraction outside the detection limits of the method and a complex refractive index input (with real part $n_{r}$ directly estimated and imaginary part $n_{i}$ adapted from literature separately for organic and inorganic components), the model produces volume scattering functions which are integrated to produce scattering and backscattering coefficients, and absorption efficiencies which are used to calculate absorption coefficients. The procedure was applied to PSDs and PRIDs derived from natural samples retrieved in UK coastal waters and analysed using a CytoSense flow cytometer (CytoBuoy b.v., Netherlands). Optical closure analysis was carried out between reconstructed IOPs and in situ IOPs measured using an ac-9 spectrophotometer and a BB9 backscattering meter (WET Labs Inc., OR) in the same waters. The procedure is shown to achieve broad agreement with particulate scattering $\left(b_{p}\right)$ and backscattering $\left(b_{b p}\right)$ (RMS\%E: $35.3 \%$ and $44.5 \%$ respectively) and to a lesser degree with backscattering ratio $\left(\widetilde{b}_{b p}\right)$ (RMS\%E: 77\%). The procedure however generally overestimated particulate absorption $\left(a_{p}\right)$ (RMS\%E: 202.3\%). This degree of closure was dependent on applying recently developed scattering error corrections to both absorption and attenuation in situ measurements. Not only do these results indirectly validate the FC method as a useful tool for PSD and PRID determination in natural particle populations, they also suggest that Mie theory may be a sufficient model for bulk IOP determination, with previously reported difficulties potentially being caused by inadequately corrected IOP measurements. Finally, in a feature unique to the FC method, the concurrent size and refractive index retrieval enabled assessment of the relative contributions that organic vs. inorganic, fluorescent vs. non-fluorescent fractions of the particle populations had on the IOPs, and identified which size classes had the largest influence on each of these properties. (C) 2017 Optical Society of America
\end{abstract}

OCIS codes: (010.1030) Absorption; (010.4450) Oceanic optics; (120.4640) Optical instruments; (290.4020) Mie theory; (290.5850) Scattering, particles; (350.4990) Particles.

http://dx.doi.org/10.1364/AO.99.099999

\section{INTRODUCTION}

The optically significant constituents of seawater are often characterized using a small number of proxy material types e.g. phytoplankton, detritus and coloured dissolved organic material. In reality, each of these proxies contains sub-populations that would potentially be of great interest if it were possible to separate their contributions to bulk optical signals. In practice there is limited scope to experimentally partition inherent optical properties (IOPs) to e.g. species level. For example, chemical processing of filter pad absorption enables partitioning of particulate absorption into components associated with pigments and a remainder that is typically associated with detritus [2,3]. It is currently not feasible to achieve even this level of partitioning experimentally for scattering and backscattering data.

In contrast, flow cytometry (FC) provides very rapid analysis of individual particles and ability to characterize concentrations of subpopulations within mixed natural samples. In ocean science, the focus has traditionally been on using this technology to examine biological particles, primarily algal populations, with fluorescence signals used to trigger data collection and particle sizing limited at the low end by laser beam width. Ackleson \& Spinrad and Green et al. $[4,5]$ pioneered an approach to extract additional size and refractive index information for small particles using a Mie inversion scheme. This approach has seldom been adopted since, potentially because it has been difficult to scale up to bulk observations such as IOPs and remote sensing signals. 
More recently, Davies et al. [6] have shown that the size class (submicron to $\sim 10 \mu \mathrm{m}$ ) that the Ackleson \& Spinrad approach provides is also responsible for the bulk of scattering and backscattering under typical oceanic conditions where the particle size is well characterized by a Junge distribution. At roughly the same time, there have been significant improvements in correction methods for in situ IOP measurements, with McKee et al. [7] providing a means to correct both absorption and attenuation measurements for scattering collection angle errors. These have been shown to be particularly significant for attenuation signals and subsequently for estimates of scattering coefficient, with corrected values being as much as a factor of two greater than original, uncorrected data.

This level of potential error in measured scattering data raises the intriguing possibility that previous attempts to relate particle size distributions to bulk optical signals using Mie theory may have failed at least in part due to limitations in the quality of the scattering data used to test the quality of modelled values.

The Ackleson-Green Mie-inversion approach to flow cytometry has recently been revisited by Duforêt-Gaurier et al. and Moutier et al. [8,9], with significant success in determining forward, sideward and backward scattering cross sections in the case of polystyrene standard beads and of two morphologically different phytoplankton species, respectively. In addition, Agagliate et al. [1] have presented a new interpretation of the technique, and demonstrated successful closure between estimated and expected size and refractive indices in various suspensions of polystyrene standard beads and oil droplets (within the sub-micron to $\sim 10 \mu \mathrm{m}$ operational range), and between flow cytometry-derived and independent particle size distribution (PSD) estimates using in situ LISST instruments (Sequoia Scientific Inc.) for a set of natural seawater samples, at least in terms of PSD slope and other major features.

Building on these recent successes, the aim of this study is to assess the extent to which the PSDs and particle real refractive index distributions (PRIDs) produced by the FC approach can be used to reconstruct bulk IOPs using Mie forward modeling. For this to be successful, several key factors would have to come together: 1. PSDs and PRIDs from the FC approach (with suitable extrapolation) need to reflect optically relevant characteristics of natural particle populations; 2 . The IOPs used to assess the performance of the modelling need to be both sufficient and consistent with the modelling approach i.e. take account of angular collection limitations of the sensors and correct appropriately; and 3. Mie theory has to adequately capture the scattering characteristics of randomly oriented, geometrically complex natural particle populations. As well as potentially revealing fundamentally important understanding of the mutual consistency of several measurement and modelling approaches, success here would validate the PSDs and PRIDs derived from flow cytometry. Furthermore, this would potentially open a new route to establish the contribution of sub-populations of particles to bulk IOPs, including scattering signals; indeed, only very few alternatives are currently available for this, e.g. Zhang et al. [10]. The knowledge of bulk IOPs is important to understand the marine environment, as they inform radiative transfer models, primary production models and ocean colour remote sensing algorithms. The ability to partition these bulk values into individual contributions would further this understanding, and may eventually be scaled up to relate remote sensing signals to specific particle classes.

Two IOPs are fundamental in the sense that all others can be derived from them: the spectral absorption coefficient, $a$, and the spectral volume scattering function (VSF), $\beta$. The spectral absorption coefficient $a$ represents the wavelength-dependent fraction of light absorbed within a unit volume per unit of distance travelled in the medium, where $\lambda$ is the wavelength. The spectral volume scattering function $\beta$ represents the wavelength-dependent polar angular distribution of scattered intensity per unit of incident irradiance per unit volume, i.e. how much light is scattered into each angle, in bulk, by the water, with the angle ranging from zero to $\pi$ radians (where zero is the direction of the incident light). The most important physical properties of the particle population to affect particulate IOPs are the size and complex refractive index of the particles. These are however also difficult to accurately determine over the entire range of optically relevant sizes.

The complex refractive index $(n)$ is at the core of scattering and absorption. Light is scattered at the interface between mediums with differing real parts of the refractive index $\left(n_{r}\right)$, while absorption of light is determined by the imaginary part of the refractive index $\left(n_{i}\right)$ of a medium. At the same time, the complex refractive index of particles is difficult to determine in the case of mixed populations: in most cases experimental results produce bulk values for a whole assemblage of particles, and most literature values are given for monotypic particle suspensions or provide an average for a whole population. Relevant examples of the methods used in literature for real refractive index retrieval include techniques such as immersion of particles in various media until scattering disappears [11,12]; bulk derivation from volume scattering functions and size distributions [13,14]; and bulk derivation from attenuation and absorption efficiencies [15-17]. Further bulk methods include the algorithm developed by Twardowski et al. [18] and the anomalous diffraction method $[19,20]$ which has also the advantage of calculating a value for the imaginary part of the refractive index. Values for the imaginary part of the refractive index are otherwise quite sparse in literature, e.g. [21,22].

Information about the size of the particles present in a suspension is conveyed as a particle size distribution. PSDs represent the concentration of particles within each size class of the particle population. These classes are somewhat arbitrary subdivisions based on a definition of "size", which can be variably represented through volume, equivalent area or relevant length of each particle. The choice is most often guided by either the context of the study, the shape of the particles or the nature of available measurements. PSDs are difficult to determine completely due to the wide range of optically relevant sizes, spanning from tens of nanometres to a few millimetres [6,23]. Most studies rely on some parametrization of the PSD, of which the most common form in the case of natural waters is the power law or Jungian distribution [24], adopted following studies which found small particles far outnumber large particles in the sea [25-27]. A power law distribution is an open-ended, logarithmically linear spectrum, with illdefined average size and average concentration always close to that of the smallest size considered. Other techniques involve the reconstruction of PSDs, either via inversion of the volume scattering function, e.g. using laser diffractometers such as the LISST series of instruments [28-30] which use Mie theory to calculate the scattering kernel relating energy received by the detectors to the number density of particles, or by iterative reconstruction of the measured VSF via modelled contribution of log-normal particle populations [10]. Overall, availability and reliability of PSD and RI determinations are still largely open to improvement, despite their fundamental influence on optical properties.

In recent work carried out on natural water samples obtained during a research cruise in UK coastal waters (UKCW dataset), a flow cytometric method based on the original approach by Ackleson \& Spinrad [4] was developed to provide particle by particle estimation of size and real refractive index, allowing for the determination of organic, inorganic, and fluorescent fractions within the total particle population and the assessment of the relative prevalence of particle sizes within each [1]. By simulating scattering within the flow cytometer through Mie theory and establishing a correspondence between real and 
simulated particles through standards of known size and refractive index, this FC method converts flow cytometric forward scattering (FWS) and side scattering (SWS) values into particle diameters and real refractive indices, providing PSDs retrieved by direct assessment of particle size and, uniquely, particle refractive index distributions. Similarly to what PSDs do with particle size, these PRIDs represent the concentration of particles within each real refractive index class of the particle population. The research cruise (HE442) took place between the 4th and 21st of April 2015 on board the RV Heincke, and consisted of sixty-two stations sampled across a variety of Case 1 and Case 2 waters around the coast of the UK (Fig. 1). This resulted in a total of 50 natural water samples with complete sets of FC data and matching data from other instruments. This included backscattering, attenuation and absorption values retrieved using an ac-9 absorption and attenuation meter and a BB9 backscattering meter.

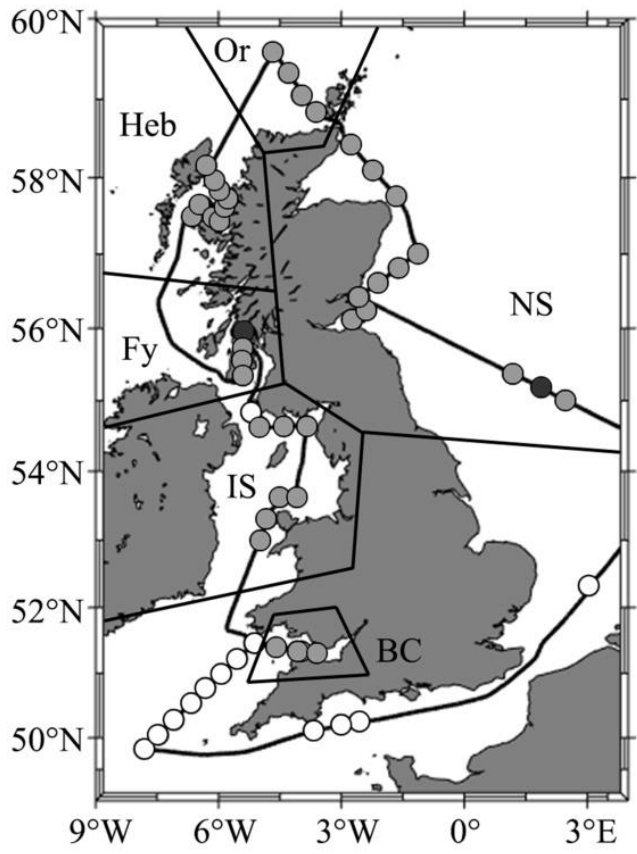

Fig. 1. Track of the HE442 research cruise, which took place in April 2015 in UK coastal waters aboard RV Heincke. Out of the 62 measurement stations visited a total of 50 complete sets of data were retrieved, matching flow cytometric data and ancillary measurements (light grey circles). Dark grey circles denote stations where two samples were taken. The dataset was divided into area groupings to highlight regional behaviour in the metadata and the measured IOPs. In clockwise order: Bristol Channel (BC), Irish Sea (IS), Loch Fyne and Firth of Clyde (Fy), Hebrides and Skye (Heb), North Atlantic - Orkneys (Or) and North Sea (NS).

In this study, optical models of scattering were coupled to the UKCW dataset, making the reconstruction of bulk IOP estimates and, more importantly, of the fractional contribution of each particle subpopulation to total IOPs possible. Since the FC-determined PSDs only cover part of the optically relevant diameter range $0.05-2000$ $\mu \mathrm{m}$ ) [6], extrapolations were needed to account for the particle fraction outside the detection limits of the FC method. Complex refractive indices were then formed by combining the directly estimated PRIDs with imaginary refractive indices adapted from literature for both organic and inorganic components [22]. Once the appropriate PSDs and complex refractive indices were established for the entire optically relevant particle population, a Mie forward model produced volume scattering functions which were integrated to produce scattering and backscattering coefficients, and absorption efficiencies which were used to calculate an absorption coefficient.

These modelled IOPs were validated against independent absorption, scattering and backscattering measurements, providing insight into the effects of the particle population composition on the optical properties of water. Mutual consistency would also represent a step towards optical closure, i.e. the successful agreement of modelled and/or measured parameters between independent measuring techniques. Ultimately this could extend to prediction and interpretation of remote sensing signals, with all parts founded on well-established physical principles.

\section{THEORY}

The foundation of Mie forward modelling rests on the additive nature of the inherent optical properties: all IOPs are the result of the sum of the single contributions from each of the individual constituents of a water volume. The axially symmetrical total particulate VSF in particular is expressed as

$$
\beta_{p}(\theta, \lambda)=\iint \beta_{D, n}(\theta, \lambda) N^{\prime}(D, n) d D d n,
$$

where $\beta_{D, n}(\theta, \lambda)$ is the VSF contribution of a single particle of diameter $D$ and complex refractive index $n$ and $N^{\prime}(D, n)$ is the corresponding value of the density function of the number concentration of particles. The PSDs and PRIDs determined by the FC method exist however not as density functions but as discrete collections of bins, each containing a number of particles corresponding to specific $D$ and $n_{r}$ values. Therefore, eq. (1) is more properly expressed as

$$
\beta_{p}(\theta, \lambda)=\sum_{D, n} \beta_{D, n}(\theta, \lambda) N(D, n),
$$

where $N_{D, n}$ is the total number concentration of particles within the PSD $\times$ PRID matrix bin corresponding to diameter $D$ and complex refractive index $n$ (once an imaginary component $n_{i}$ is associated to $n_{r}$ as will be described in the Methods section). By way of the results of Mie theory [31,32] eq. (2) can then be ultimately rewritten as

$$
\beta_{p}(\theta, \lambda)=\sum_{D, n} \frac{1}{2} \frac{\left(i_{1 ; D, n}(\theta)+i_{2 ; D, n}(\theta)\right)}{k^{2}} N_{D, n},
$$

where $k=2 \pi / \lambda$ is the wave number and $i_{1}(\theta)$ and $i_{2}(\theta)$ are scattering amplitude functions. The latter are the core quantities to be determined in any Mie scattering problem, and are fundamentally dependent on the diameter and refractive index of each particle [31]. The $i_{1}(\theta)$ and $i_{2}(\theta)$ functions are thus calculated for each $D$ and $n$ pair, then multiplied by the corresponding $N_{D, n}$ value, and ultimately summed over all $D$ and $n$ combinations following Eq. (3) to obtain the total particulate VSF. Finally, total particulate scattering $\left(b_{p}\right)$ and backscattering $\left(b_{b p}\right)$ coefficients are calculated by integrating the total particulate VSF over the appropriate angle ranges, i.e. $0^{\circ}-180^{\circ}$ for the former and $90^{\circ}-180^{\circ}$ for the latter (where $\theta=0^{\circ}$ indicates the forward direction). The total particulate backscattering ratio $\left(\tilde{b}_{b p}\right)$ is given simply as the ratio of $b_{p}$ and $b_{b p}$.

Mie theory can be also used to calculate the efficiency factors for attenuation and scattering, which in turn define the absorption efficiency factor through simple subtraction. The absorption efficiency factor can be then used to define the spectral absorption coefficient of a single spherical particle as

$$
a_{i}(\lambda)=\frac{\pi D_{i}^{2}}{4} Q_{a, i}(\lambda),
$$


where $D_{i}$ is the diameter of the particle and $Q_{a, i}(\lambda)$ the absorption efficiency of the particle. Analogously to eq. (2), the total particulate absorption $\left(a_{p}\right)$ is now simply defined as the sum of contributions from all particles,

$$
a_{p}(\lambda)=\sum_{D, n} a_{D, n}(\lambda) N_{D, n}=\sum_{D, n} \frac{\pi D_{i}^{2}}{4} Q_{a, i}^{D, n}(\lambda) N_{D, n},
$$

where $a_{D, n}(\theta, \lambda)$ is the absorption contribution of a single particle of diameter $D$ and complex refractive index $n$ and $N_{D, n}$ is the total number concentration of particles within the corresponding PSD $\times$ PRID matrix bin.

All Mie forward modelling calculations were handled using an independently developed MATLAB script, building upon the FASTMie code developed by Slade [33] to calculate the scattering amplitude functions $i_{1}(\theta)$ and $i_{2}(\theta)$.

\section{METHODS}

Mie-based forward optical modelling was applied to the PSDs and PRIDs determined by the FC method for the UKCW dataset of natural seawater samples, which can be found described in detail in [1]. A description of the dataset and of the measurement protocols (particularly those relative to absorption, attenuation and backscattering measurements) is summarised below. IOPs obtained by forward modelling from FC data were compared with corresponding absorption, scattering and backscattering coefficients determined by ac-9 and BB9 instruments (WET Labs Inc., OR). In all cases, $532 \mathrm{~nm}$ was used as the wavelength of choice for IOP calculations, as it is a green wavelength near the centre of the visible spectrum and one shared by both ac-9 and BB9 instruments without any need for interpolation. Resulting IOPs were then further separated into individual contributions from fluorescent/organic/inorganic fractions and from different size classes.

\section{A. Depth profiling}

Depth profiling was performed using an instrument frame equipped with Niskin bottles for sample retrieval and with both ac-9 and BB9 sensors. The frame was lowered into water at each of the stations to measure a profile, and was then kept near surface for water sampling. At a few stations, additional samples from deeper waters were taken. BB9 and ac-9 instruments logged data throughout the procedure. The samples were taken from the Niskin bottles on the frame as quickly as possible after the frame was back on deck and filled into 10-liter plastic containers. In waters with high turbidity the Niskin bottles were flushed twice to avoid settling out of particulate matter. 48 out of the 50 samples of the UKCW dataset are surface samples (max. depth: 7 $\mathrm{m}$ ), with further two samples taken from bottom depths instead.

\section{B. Flow cytometry measurement protocol}

All samples were analysed using a CytoSense flow cytometer (CytoBuoy b.v., Netherlands) once for each of four sensitivity settings of the side scattering photomultiplier tube (PMT), for 6 minutes and at a flow rate of $0.5 \mu \mathrm{L} / \mathrm{s}$. The end of each measurement was triggered after a set time rather than after the collection of a certain number of particles. The number of collected particles (typically ranging between orders of magnitude $10^{3}$ and $10^{5}$ depending on local population density and PMT setting used) thus varied from sample to sample as the instrument was left counting until the end of the allotted time. Side scattering was used as the trigger channel in all cases. Additional measurements of standard polymer beads were taken daily across the whole sampling period, and were used to calibrate the FC method by relating the resulting FWS and SWS values to the known diameters and refractive index of the standard particles. Once such relationship is established, a look-up table can be calculated to reconstruct $(D, n)$ pairs from each (FWS, SWS) combination, thus collectively providing a PSD and a PRID for each sample. A detailed description of the FC method and of the procedure followed to reconstruct PSDs and PRIDs can be found in [1], where the application of the method to the UKCW dataset is also discussed specifically.

\section{UKCW PSDs and PRIDs}

The PSDs retrieved by the FC method for the UKCW dataset broadly follow power law distributions, with the main difference between stations being the overall concentration of the particle population (Fig. 2a).
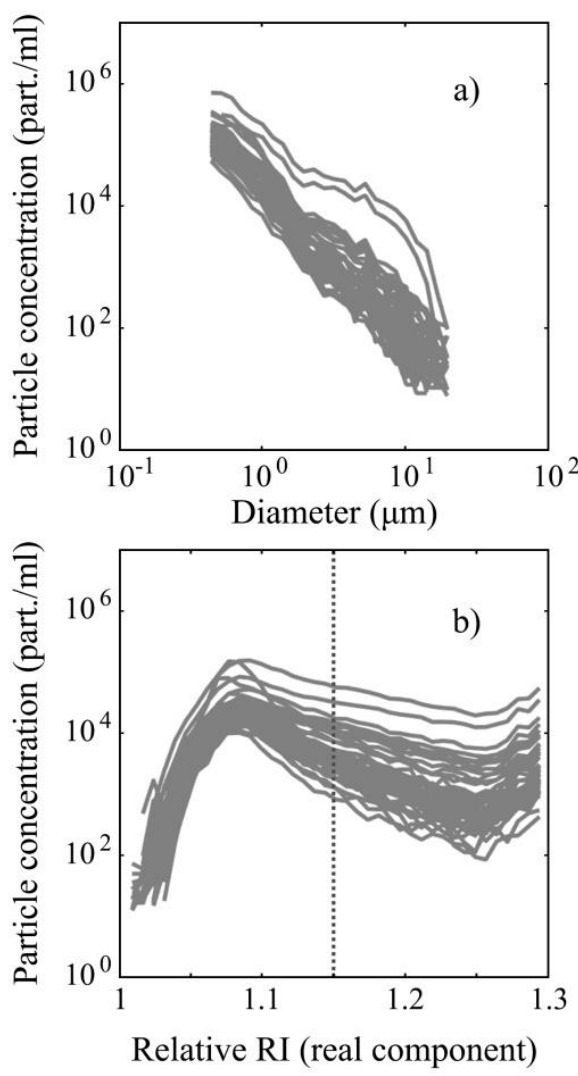

Fig. 2. Collective view of (a) all 50 UKCW PSDs and (b) UKCW PRIDs produced by the FC method. Note that real refractive index values above 1.15 (dotted line) are not precise, but still indicate high refractive indices.

Two obvious outliers are present, corresponding to samples from the turbid waters of the Bristol Channel; close inspection reveals structures that may be closer in nature to models such as the double gamma distribution proposed by Risović [34], and that indeed may be identified to a lesser degree in the other samples as well. Nonetheless, the power law approach remains a reasonable approximation for a large majority of the dataset, and was used when extrapolating the concentration of particles outside the size range of the FC method.

PRIDs retrieved by the FC method for the UKCW dataset are fairly homogeneous in shape across all samples (Fig. 2b), with distribution peaks found between 1.05-1.15 and within expectations for the $n_{r}$ values of the most common components of marine particle populations [18,35]. A tail of particles with $n_{r}>1.15$ is observed in all samples, with median concentrations 5 to 10 times lower than peak ones. Due to the difficulty of unambiguously retrieving high $n_{r}$ values 
[1], real refractive index values above 1.15 are not to be interpreted as precise, but still positively indicate high refractive indices.

\section{PSD extrapolations}

Any kind of forward modelling will require the entire optically relevant particle distribution to be included as the input, or the output will not be comparable with any independently measured bulk IOP. The FC method was found to reliably retrieve particle diameters between $\sim 0.5-10 \mu \mathrm{m}$ [1]. This range covers a large fraction of the contribution to scattering and backscattering, but the whole optically relevant range spans from tens of nanometres to a few millimetres $[6,23]$. The undetectable fraction of the particle population has to be accounted for using an approximation of the PSD to extend the range of the distribution over the whole relevant range. Ever since pioneering work in the ' 60 s and '70s found that the number of particles suspended in the ocean increased continuously and monotonically towards smaller scales [25,26], power law distributions of the type used by Junge [24] for aerosols have been the most common form of approximation for natural seawater particle populations $[27,36]$. With notable exceptions in the samples retrieved in the waters of the Bristol Channel, the PSDs determined by the FC method for the UKCW dataset broadly conformed to this model, and a least squares best fit of power law distributions was used to determine the slopes of the UKCW PSDs [1]. Consequently, following in the steps of Green et al. [37], a least squares best fit of the measured PSDs through power law distributions as defined by

$$
N(D)=N^{\prime}(D) d D=k D^{-\gamma} d D
$$

was used to extend measured PSDs as well (Fig. 3). The form given in eq. (6) is necessary because the FC PSDs have bin-like nature; accordingly, the extrapolations need to be bin-like as well.

PSDs for the UKCW dataset were extended between 0.05 and 2000 $\mu \mathrm{m}$ following Davies et al. [6]; the mean slope value of the UKCW dataset is found to be 3.35 .

\section{E. RI approximations}

Values for $n_{r}$ in the Junge extensions must be accounted for using some approximation of the PRID to extend the range of known refractive indices; various assumptions may be used to do so. The most conservative approach (and the one used in this study) is to assign a fixed value to $n_{r}$, either the same on both arms of the extension or a separate one for each. This can be done in a variety of ways (Fig. 3), e.g. by using the average $n_{r}$ of the entire measured fraction of PSD on both arms of the extension (mode A); by using the values of $n_{r}$ at the extremes of the measured fraction of PSD on the respective arms of the extension (mode B); or by using literature-derived $n_{r}$ values based on an hypothesis of probable particle composition in the extension (mode C). The results obtained using each of these different approaches will be detailed in the following. In particular, when literature values were used, 1.15 was chosen as the $n_{r}$ value of particles smaller than $1 \mu \mathrm{m}$ and 1.05 as the $n_{r}$ value representative of particles larger than $10 \mu \mathrm{m}$. A few rare instances occurred of gaps being present in some PSDs at $\sim 10$ $\mu \mathrm{m}$ when overall particle concentrations were low. Where these gaps were present in the PSDs, a value of 1.1 was used for the power law extrapolation between 1-10 $\mu \mathrm{m}$.

Since the FC method doesn't offer any information on the imaginary part of the refractive indices, $n_{i}$ values are unknown both in the available FC PSDs and in their extrapolations. Typical values for the imaginary component of the relative refractive index were therefore adapted from literature (Fig. 8 of Babin et al. [22]), for both organic and inorganic particles. These were then assigned to the particles according to the value of the real refractive index of each bin, both directly determined by the FC method and extrapolated. Accordingly, with this approach the values of the imaginary part of the refractive index in the PSD extensions will be ultimately dependent on the $n_{r}$ approximation used.

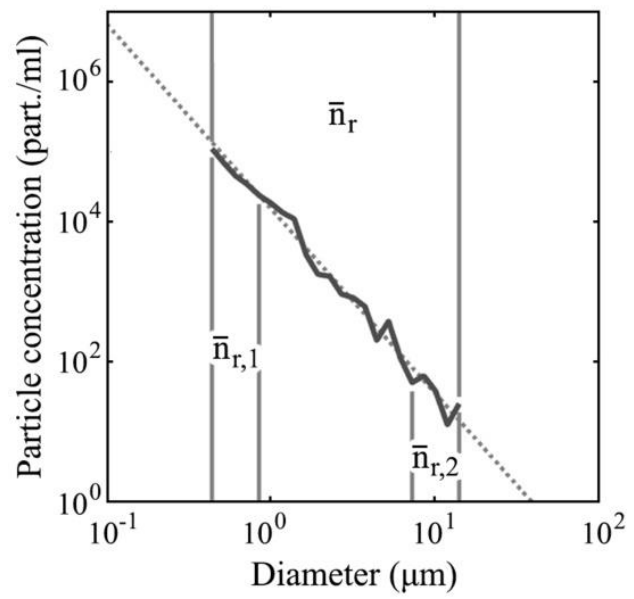

Fig. 3. Power law best fit (dotted line) and PSD extension $n_{r}$ approximations in an example of particle size distribution from the UKCW dataset (solid line). A single $n_{r}$ value averaged across the PSD range $\left(\bar{n}_{r}\right)$ may be used on both upper and lower ends of the extension (mode A), or independent $n_{r}$ values averaged over the extremes of the PSD $\left(\bar{n}_{r, 1}, \bar{n}_{r, 2}\right)$ may be used on the respective ends of the extension (mode B). Alternatively, $n_{r}$ values derived from literature based on the probable composition of the particle population in the extensions may be used (mode C).

\section{F. Attenuation and absorption measurements}

The WET Labs ac-9 instrument is a submersible, in situ absorption and attenuation meter designed to provide real-time measurements over nine wavelength channels; a detailed description of the instrument is given by Twardowski et al. [38]. The ac- 9 absorption tube uses total internal reflectance from its internal glass wall to redirect scattered light towards the absorption sensor and minimize scattering losses. However, scattering corrections are still required to account for residual losses. Furthermore, the attenuation sensor collects photons scattered in forward directions at angles smaller than the attenuation sensor aperture, which is a lens-pinhole with collection angle $\theta_{c}=0.9^{\circ}$. This artificially lowers attenuation values. A number of different methods for scattering correction of absorption exist, of which the most commonly used has traditionally been the proportional correction [39]. In recent years, two new correction procedures have been proposed, the semi-empirical correction [40] and the iterative correction [7]. The iterative correction in particular corrects for errors in both absorption and attenuation measurements.

The ac-9 attenuation and absorption meter was operated following methodologies established in the user's manual and protocol for this instrument [41,42]; a detailed description of measurement procedures can also be found in Lefering et al. [43]. Attenuation and absorption spectra represent medians of the time series recorded while the frame was kept at a certain depth for sample collection. This minimised mismatch between in situ IOP data and samples analysed using the FC method. In situ data were scattering-corrected using the iterative correction proposed by McKee et al. [7], and were further corrected for salinity and temperature dependence following Pegau et al. [44]. Particulate absorption was finally determined by subtraction of the contribution of CDOM (coloured dissolved organic matter) to total 
absorption, as determined using the long path-length liquid waveguide capillary cell technique (LWCC) [45].

\section{G. Backscattering measurements}

The WET Labs BB9 instrument is a submersible, in situ backscattering sensor which similarly to the ac-9 is designed to provide real-time measurements over nine wavelength channels. A thorough review and description of the principles behind backscattering measurements can be found in Sullivan et al. [46]. In essence, modern backscattering sensors like the BB9 are VSF meters. They take advantage of a peculiarity of scattering phase functions, which are observed to have low variability in their shape at backwards angles [47], leading to the determination of conversion factors capable of providing estimates of backscattering $b_{b}$ values from single measurements of the VSF at backward angles, known as $\chi$ factors $[48,49]$. BB9 backscattering measurements need to be corrected for absorption over the pathlength of the beam used to illuminate the sample. This makes concurrent ac-9/BB9 measurements important.

As with the ac-9, the BB9 was operated following procedures established in the user's manual for the instrument [50]. Backscattering data was corrected for pathlength absorption using ac9 absorption data, itself scattering-corrected using the proportional correction [39] and averaged over the measurement period. In turn, the BB9 backscattering data was used to inform the iterative correction procedure used for ac- 9 absorption and attenuation, which explains why pathlength absorption was corrected using ac-9 data corrected with the proportional rather than the iterative method.

\section{RESULTS}

Tab. 1 presents the results of the comparison between IOPs as determined by Mie forward modelling for the UKCW dataset and IOP values measured by ac- 9 and BB9 instruments. Mode B (independent $n_{r}$ values averaged over the extremes of the PSD ) was found to provide the best overall retrieval of IOPs among the three modes of $n_{r}$ approximation in the PSD extensions, and corresponding results are shown in Fig. 4; all further results presented in the following correspond to this $n_{r}$ approximation as well. The quality of the agreement was evaluated as the root mean square percentage error (RMS\%E) calculated over the differences between modelled and measured values (i.e. compared to the 1:1 line). Retrieval of IOPs was found to be variable, with trends to either underestimate or overestimate the values retrieved by ac- 9 and BB9. Nonetheless, overall agreement is good for particulate scattering and backscattering
(RMS\%E: $35.3 \%$ and $44.5 \%$ respectively), with the particulate backscattering ratio demonstrating lower agreement as expected due to the negative impact of compounding errors by taking ratios (RMS\%E: 77\%). Modelled absorption values generally overestimated ac-9 particulate absorption (RMS\%E: 202.3\%): while the agreement here is poor, absorption was expected to be the least successful among the 4 reconstructed IOPs due to its reliance on crude approximations for $n_{i}$ values. Varying agreement of FC and in situ IOPs can to some extent be attributed to uncertainties in the in situ determinations. Recent work by Lefering et al. [43], however, demonstrates the high quality of IOP measurements by the largely successful reconstruction of photosynthetically available radiation (PAR) and remote sensing reflectance $\left(R_{r s}\right)$ values through radiative transfer models informed by ac-9 and BB9 values.

When considering the sample-by-sample ratio of modelled FC IOPs vs. measured IOPs, different behaviours were observed for each of the IOPs. FC absorption displayed large variance across the entire dataset (Fig. 5a), while FC scattering was generally lower than ac-9 scattering, with disagreement progressively increasing in later samples (Fig. 5b). This could represent a breakdown in the performance of the ac-9 iterative correction used to correct ac- 9 data under algal bloom conditions, possibly as a consequence of deviations from the FournierForand phase function that underpins the method [7].

\begin{tabular}{cccc}
\cline { 2 - 4 } & \multicolumn{3}{c}{ RMS\%E } \\
\hline IOP & Mode A & Mode B & Mode C \\
\hline Absorption & 199.5 & 202.3 & 209.7 \\
Scattering & 34.3 & 35.3 & 35 \\
Backscattering & 60.1 & 44.5 & 48.9 \\
Backscattering ratio & 112.7 & 77 & 94.3 \\
\hline
\end{tabular}

Tab. 1. Agreement between modelled and measured IOPs for the UKCW dataset for each of the three modes of $n_{r}$ approximation (see section 3.E), expressed as root mean square percentage errors (RMS\%E) given relative to the 1:1 line. Mode A: a single $n_{r}$ value averaged across the PSD range $\left(\bar{n}_{r}\right)$ is applied to both upper and lower ends of the power law extrapolation. Mode B: independent $n_{r}$ values averaged over the extremes of the PSD $\left(\bar{n}_{r, 1}, \bar{n}_{r, 2}\right)$ are applied to the respective ends of the power law extrapolation. Mode C: $n_{r}$ values derived from literature and based on the probable composition of the particle population a e applied to the power law extrapolation. 

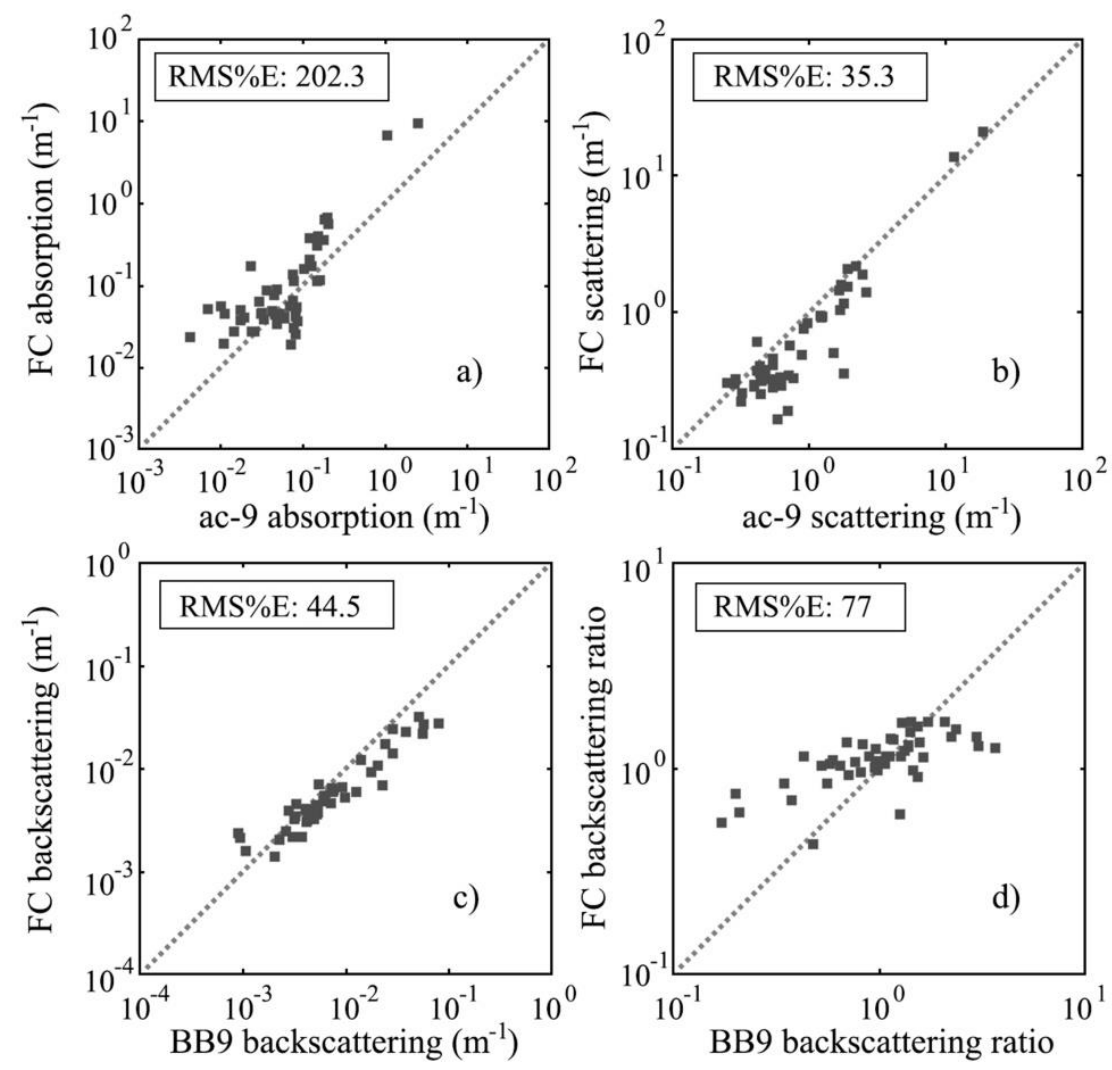

Fig. 4. Optical closure analysis of the UKCW dataset for Mode B of $n_{r}$ approximation in the Junge extensions for (a) absorption, (b) scattering, (c) backscattering and (d) backscattering ratio.
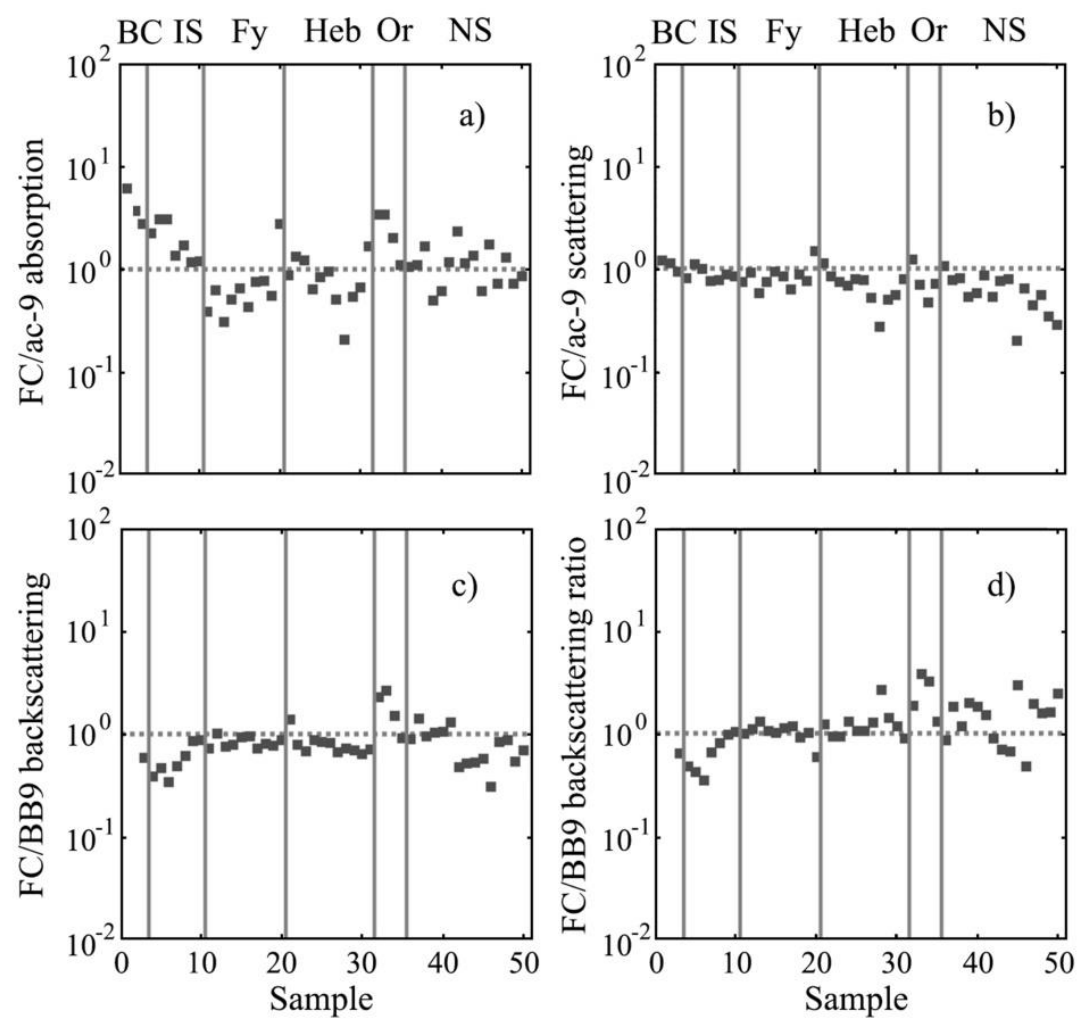

Fig. 5. FC vs. in situ IOP ratios for (a) the particulate absorption coefficient, (b) the particulate scattering coefficient, (c) the particulate backscattering coefficient and (d) the particulate backscattering ratio. The vertical lines reflect the regional groupings presented in Fig. 1. Note that the first two data points for BB9 backscattering and in situ backscattering ratio are missing because of saturation in the BB9 backscattering meter. 

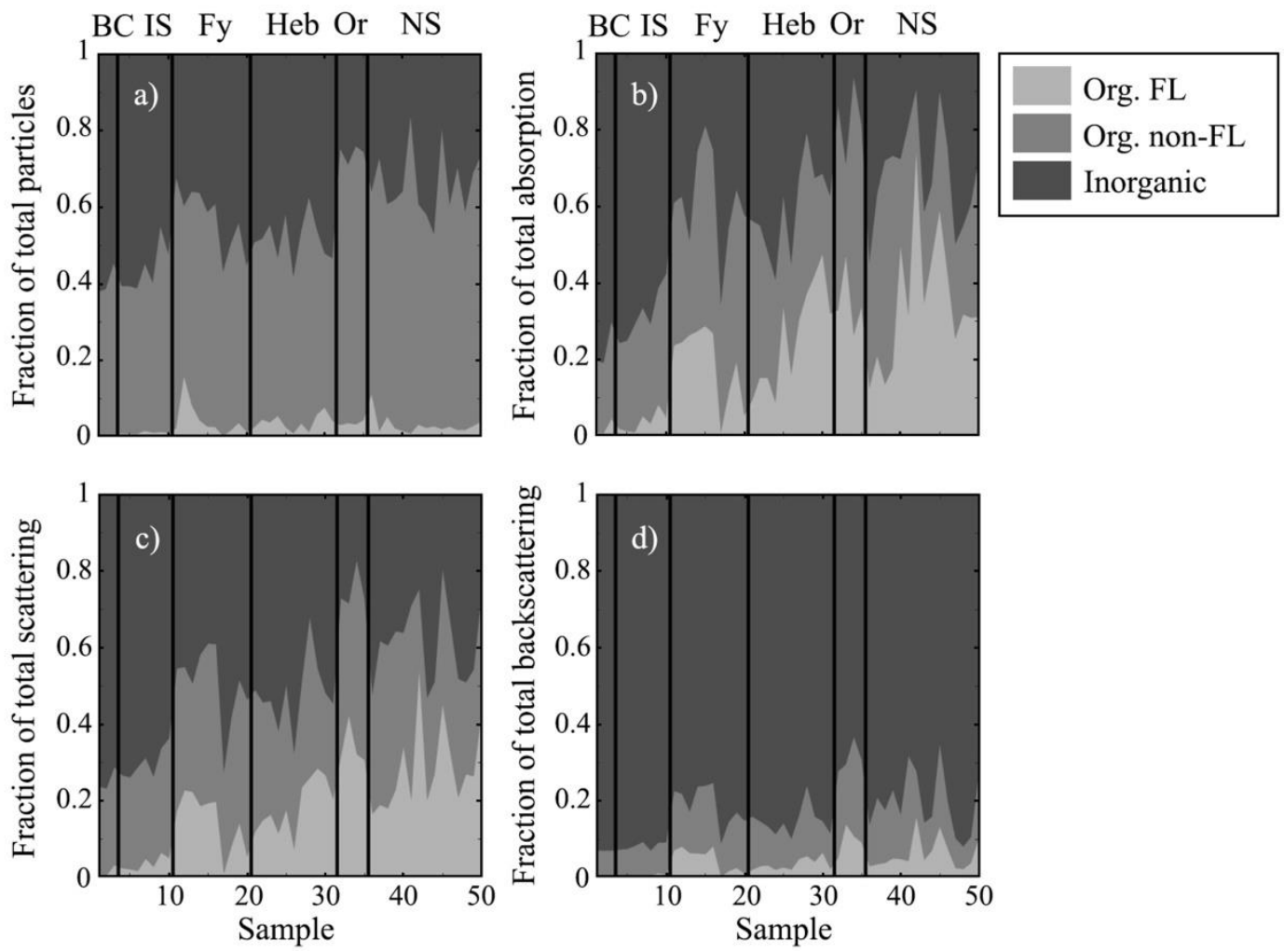

Fig. 6. (a) Relative abundance of the inorganic and organic (fluorescent and non-fluorescent) fractions of the total particle populations, and contribution of each of these population fractions to (b) total absorption, (c) scattering and (d) backscattering coefficients. The vertical lines reflect the regional groupings presented in Fig. 1.

FC backscattering was generally lower than BB9 backscattering, with larger disagreement in the Irish Sea, Orkneys and North Sea samples (Fig. 5c). FC backscattering ratios (Fig. 5d) generally replicated the pattern displayed by the backscattering data (Fig. 5c). For $b_{p}, b_{b p}$ and $\widetilde{b}_{b p}$ samples from the Firth of Clyde and the Hebrides were seen to produce results which were consistently closer to the validation IOPs.

The capability of the FC method to determine the size and real refractive index of each particle, combined with the ability to detect fluorescence, enabled analysis of the individual contribution of fluorescent and non-fluorescent organic and inorganic fractions of the particle population to the IOPs, although limited to the effective size detection range of the method. Across the 50 samples in the UKCW dataset, inorganic particles were seen to account for particle population fractions ranging from $16.6 \%$ to $62.2 \%$ of the total, with an average value of $43 \%$ (Fig. 6a). Samples from the Bristol Channel and the Irish Sea displayed the largest inorganic content, while later samples collected west of Orkney and from the North Sea were generally dominated by organic particles. Fluorescent organic particles were found to account for a small fraction of the particle population $(0.1-15.6 \%)$, lower than $5 \%$ in most samples. The organic fraction was seen to have a strong influence on both absorption and scattering, with fraction contributions between $18.9-93.7 \%$ and $23-82.6 \%$ of the total respectively across the UKCW dataset (Fig. 6b-c). Lowest organic contributions came from the Irish Sea and (particularly) from the Bristol Channel. Fluorescent organic content was also observed to have significant impact on $a_{p}$ and $b_{p}$ despite its small population fraction, with contribution values as high as $73.9 \%$ and $53.8 \%$ respectively and generally higher than $20 \%$ in a large number of samples. Once again, the Irish Sea and Bristol Channel samples (where the fluorescent content was found to be numerically negligible) displayed the lowest fluorescent fraction contribution to both IOPs. In contrast, backscattering was found to be largely dominated by the inorganic fraction of the particle population, with values ranging from $63.3 \%$ to 93.1\% of the contribution (Fig. 6d).

The reason behind the different observed fractional IOP contributions rests in the different response that the IOPs have to particles of the same size, as demonstrated when the individual IOP contribution of the measured FC fraction of the PSD is compared to those induced by the upper and lower PSD extensions (i.e. large and small particles respectively). Particulate absorption (Fig. 7a) was found to be dominated by large particles above $\sim 10 \mu \mathrm{m}(27.1-98.1 \%)$, with only minor contribution from particles smaller than $\sim 0.5 \mu \mathrm{m}(0.1-$ 12.1\%). Particulate scattering (Fig. 7b) was found to be overwhelmingly influenced by particles larger than $\sim 0.5 \mu \mathrm{m}$ (92.1$99.9 \%$ of the contribution), with a non-negligible contribution from particles larger than $\sim 10 \mu \mathrm{m}$ (3.3-80.6\%). Finally, backscattering $b_{b}$ (Fig. 7c) was found to be largely influenced by particles smaller than $\sim 10 \mu \mathrm{m}$ (53.4-99.4\% of the contribution), with a sizeable influence from particles smaller than $\sim 0.5 \mu \mathrm{m}(1.4-49.9 \%)$.

Considering these results, the large inorganic contribution to the total backscattering shown in Fig. 6d may be interpreted to suggest that small particles in the UKCW dataset were mostly of inorganic nature; it should be noted however that the lower diameter detection limit of the FC method is slightly higher for organic particles than it is for inorganic particles, because given equal diameters a higher refractive index produces a higher side scattering signal [1]. Therefore, organic number densities fall off slightly earlier than inorganic as diameters approach the detection limit. Although all care was taken to minimise this spurious effect, it is likely that some part of the $b_{b p}$ fraction of Fig. $6 \mathrm{~d}$ was caused by it. 

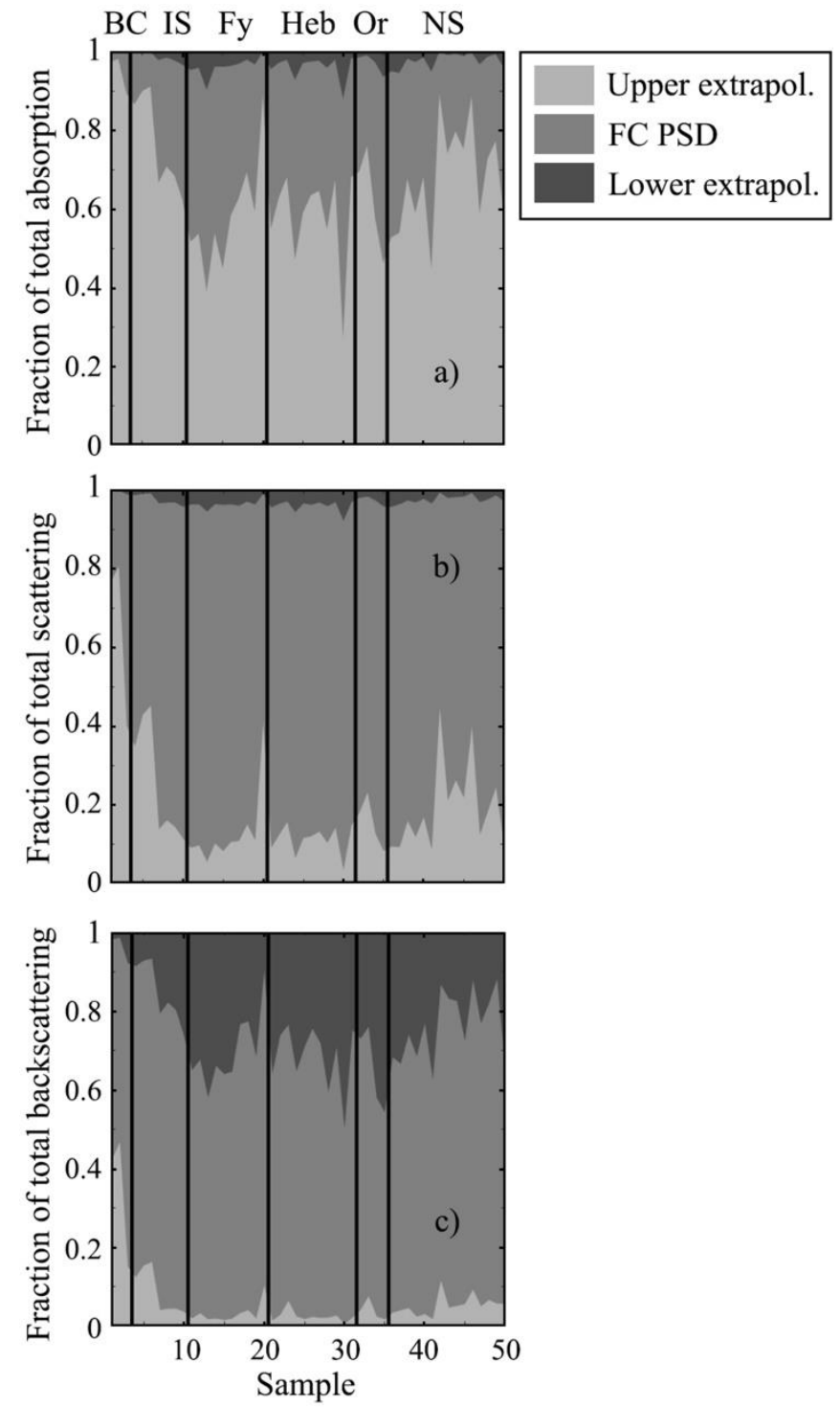

Fig. 7. Comparison of the relative contributions of the FC PSD and of its upper and lower power law extensions (i.e. large and small particles respectively) to (a) total absorption, (b) scattering and (c) backscattering coefficients. The vertical lines reflect the regional groupings presented in Fig. 1.

\section{DISCUSSION}

The best results for the closure analysis between measured and modelled IOPs for the UKCW dataset were achieved for particulate scattering and backscattering coefficients. In the latter case, more refined approximations for the $n_{r}$ values used in the Junge extensions of the PSDs (i.e. $n_{r}$ averages based on available data as opposed to fixed values adapted from literature) markedly improved the quality of the agreement, an effect consequently also reflected in backscattering ratio values. Agreement between FC and ac-9 particulate absorption coefficients remained the least successful across all three $n_{r}$ approximations used, perhaps as expected for a quantity which is chiefly influenced by imaginary refractive indices. The FC method of flow cytometric determination of size and refractive index offers no information on the imaginary component of the refractive index, so that no better solution than adapting literature values to the organic/inorganic fractions of the particle population could be used.
Given the crude nature of such an approximation, the absorption modelling procedure is likely to benefit from an optimization algorithm designed to identify optimal imaginary refractive index values. Future development of libraries of $n_{i}$ data for different species could also be usefully applied to this type of analysis by combining with the species characterization capabilities that flow cytometry provides.

Given the relatively narrow size range that the FC method addresses, the need to extrapolate both size and refractive index values, and the absence of direct observations of $n_{i}$, the degree to which forward modelled IOPs match measured values is surprisingly good. Variation in the quality of match-ups across the dataset can be attributed to a number of interwoven factors. For example, selection bias in favour of small particles potentially affects absorption retrieval more than scattering and backscattering, as Fig. 7a demonstrates the relatively strong contribution from the upper extrapolation. Fig. 5 indicates that there may be regional factors at play, probably corresponding to changes in the composition of the particle population. It is worth noting that it is quite likely that such changes might also influence the performance of scattering correction procedures for ac-9 absorption and attenuation measurements, and therefore influence the degree of agreement between the measured IOPs and IOPs derived from Mie forward modelling. There is scope to extend the FC sampling procedure to capture a wider range of particle sizes at the high end and to improve statistical significance throughout.

The long string of assumptions and calculations steps required to derive modelled IOPs is bound to introduce large uncertainties in the final values, with the largest effect on absorption for which only crude $n_{i}$ approximations were employed, potentially explaining the high $\mathrm{RMS} \% \mathrm{E}$ for the absorption match-up to some extent However, it is clear that the current iteration of the FC method already provides a useful insight into the contribution of different particle components to the formation of bulk particulate IOPs. The overall broad agreement between measured and modelled IOPs provides useful validation of the PSDs and PRIDs generated by the FC method. In doing so, it also suggests that Mie theory is sufficient to provide useful estimates of bulk IOPs for natural particle populations. This is not to say that Mie theory is capable of accurately predicting optical properties for all marine particles; indeed, there are well-established situations where more complex optical models are required, especially in the case of samples dominated by phytoplankton: Clavano et al. [51] offer an in-depth overview of this issue. Rather, results indicate that Mie theory, which is analytical, conservative and computationally fast, has the ability to reproduce bulk IOPs for randomly orientated, mixed populations of naturally occurring marine particles. A possible interpretation of such results would be to suggest that the nature of the $\mathrm{FC}$ method produces diameter and $n_{r}$ values that correspond to spheres optically equivalent to the particles processed by the flow cytometer; the modelled IOPs therefore would be compatible with bulk IOP measurements taken by instruments which observe ensembles of randomly orientated particles. However, more data is required to substantiate this interpretation.

It is important to consider that in situ IOPs used to validate the forward modelling are themselves subject to variable degrees of error. Forward modelled scattering values, resolved down to $0.01^{\circ}$ in the forward direction, demonstrated mutual consistency with in situ values generated using iterative correction of the scattering error in the ac-9 attenuation measurements. This particular correction was chosen specifically because other correction methods do not include a correction of attenuation coefficients, thus affecting scattering coefficient retrieval. Similar degrees of closure with uncorrected in situ $b_{p}$ data is possible by restricting calculations to angles beyond the transmissometer collection angle, but are no better in quality. This is good circumstantial evidence that the iterative ac-9 scattering 
correction is a useful development. On the other hand, as mentioned previously, the iterative correction relies on selection of appropriate scattering phase functions and there is evidence (Fig. 5b) that there is possibly some degree of breakdown in performance as waters become increasingly dominated by large phytoplankton [43].

Finally, it is important to stress that the FC method and the IOP forward modelling based on its results are not proposed as techniques capable of replacing any of the already established techniques for IOP measurements, but rather as tools to assist and complement those other techniques. Furthermore they are presented as a viable method for the estimation of the individual contributions from different particle size classes and particle types to the total particulate IOPs. The ability to partition bulk IOPs by size and/or particle type demonstrated in Fig. 6-7 offers a unique capability to explore the contribution of subpopulations of particles to optical signals in the ocean. Ultimately this could be scaled up to understanding the relative contribution of subpopulations to ocean colour remote sensing signals and the long cherished concept of relating satellite data to individual particle observations could become a reality.

\section{CONCLUSION}

PSDs and PRIDs determined using the FC method for natural seawater samples were used as inputs to Mie forward optical modelling. With the selection of appropriate PSD extrapolations and $n_{r}$ approximations, which accounted for undetected particles, the calculations produced IOP values which were then compared with corresponding measured IOPs. UKCW dataset IOP match-up results were found to be variable, reflecting geographic variability and possibly limitations in the quality of in situ IOPs used for validation. However, given the relatively narrow $\mathrm{FC}$ method size detection range, the need to extrapolate PSDs and approximate $n_{r}$ values to account for undetected particles, and an inherent lack of direct $n_{i}$ measurements, the degree of agreement between modelled and measured IOPs is surprisingly good. These results validate the PSDs and PRIDs produced by the FC method, suggest a reassessment of the value of Mie theory in the context of predicting bulk IOPs for natural particle populations and underline the requirement to consider the role of measurement uncertainties, particularly systematic errors, when using in situ IOPs for validation of forward optical modelling.

Finally, PSDs and PRIDs as determined by the FC method offered the chance to gain useful insight into the individual contributions of different particle subpopulations to the bulk IOPs of seawater samples. By further associating models of particle density and of organic carbon and chlorophyll content to the PSDs and PRIDs provided by the FC method, future work may find the potential to similarly explore the biogeochemistry of natural mixed particle populations, perhaps providing the means to bridge the divide between optical and biogeochemical properties of natural particles.

Funding Information. The HE442 cruise with RV Heincke was conducted under the grant number AWI-HE442. Lefering and Agagliate received funding from the MASTS pooling initiative (The Marine Alliance for Science and Technology for Scotland). MASTS is funded by the Scottish Funding Council (grant reference HR09011) and contributing institutions.

Acknowledgment. The authors wish to thank the captain and the crew of RV Heincke for their support and help during the HE442 research cruise. Mike Twardowski and Rüdiger Röttgers are thanked for very helpful conversations about this data set. MASTS funding support is gratefully acknowledged.

\section{References}

1. J. Agagliate, R. Röttgers, M. S. Twardowski, and D. McKee, "Evaluation of a flow cytometry method to determine size and real refractive index distributions in natural marine particle populations," Submitted paper (2017).

2. A. Bricaud and D. Stramski, "Spectral absorption coefficients of living phytoplankton and nonalgal biogenous matter: a comparison between the Peru upwelling area and the Sargasso Sea," Limnol. Oceanogr. 35(3), 562-582 (1990)

3. I. Lefering, R. Röttgers, R. Weeks, D. Connor, C. Utschig, K. Heymann, and D. McKee, "Improved determination of particulate absorption from combined filter pad and PSICAM measurements," Opt. Express 24, 24805 24823 (2016).

4. S. G. Ackleson and R. W. Spinrad, "Size and refractive index of individual marine particulates: a flow cytometric approach," Applied Optics 27, 1270 (1988).

5. R. E. Green, H. M. Sosik, R. J. Olson and M. D. DuRand, "Flow cytometric determination of size and complex refractive index for marine particles: comparison with independent and bulk estimates," Applied Optics 42, 526 (2003).

6. E. J. Davies, D. McKee, D. Bowers, G. W. Graham and W. A. M. NimmoSmith, "Optically significant particle sizes in seawater," Applied Optics 53, 1067 (2014).

7. D. McKee, J. Piskozub, R. Röttgers and R. A. Reynolds, "Evaluation and Improvement of an Iterative Scattering Correction Scheme for in situ Absorption and Attenuation Measurements," Journal of Atmospheric and Oceanic Technology 30, 1527-1541 (2013).

8. L. Duforêt-Gaurier, W. Moutier, N. Guiselin, M. Thyssen, G. Dubelaar, X. Meriaux, L. Courcot, D. Dessailly, and H. Loisel, "Determination of backscattering cross-section of individual particles from cytometric measurements: a new methodology," Opt. Express 23, pp. 31510-31533 (2015).

9. W. Moutier, L. Duforêt-Gaurier, M. Thyssen, H. Loisel, X. Mériaux, L. Courcot, D. Dessailly, and S. Alvain, "Scattering of individual particles from cytometry: tests on phytoplankton cultures," Opt. Express 24, pp. 2418824212 (2016).

10. X. Zhang, M. Twardowski and M. Lewis, "Retrieving composition and sizes of oceanic particle subpopulations from the volume scattering function," Applied Optics 50, 1240 (2011).

11. W.C McCrone, R.G. Draftz, and J. G. Delly, The Particles Atlas (Ann Arbor Science Publ., 1967).

12. F. D. Bryant, B. A. Seiber and P. Latimer, "Absolute optical cross sections of cells and chloroplasts," Archives of Biochemistry and Biophysics 135, 97-108 (1969).

13. H. R. Gordon and O. B. Brown, "A theoretical model of light scattering by sargasso sea particulates," Limnology and Oceanography 17, 826-832 (1972).

14. J. R. V. Zaneveld, D. M. Roach and H. Pak, "The determination of the index of refraction distribution of oceanic particulates," Journal of Geophysical Research 79, 4091-4095 (1974).

15. A. Bricaud, A.-L. Bédhomme and A. Morel, "Optical properties of diverse phytoplanktonic species: experimental results and theoretical interpretation," Journal of Plankton Research 10, 851-873 (1988).

16. D. Stramski and D. A. Kiefer, "Optical properties of marine bacteria." In R. W. Spinrad. (ed.), Ocean Optics X. Proceedings 16-18 April, Orlando, Florida (SPIE, 1990) 1302, 250-268.

17. D. Stramski and A. Morel, "Optical properties of photosynthetic picoplankton in different physiological states as affected by growth irradiance," Deep Sea Research Part A. Oceanographic Research Papers 37, 245-266 (1990).

18. M. S. Twardowski, E. Boss, J. B. Macdonald, W. S. Pegau, A. H. Barnard and J. R. V. Zaneveld, "A model for estimating bulk refractive index from the optical backscattering ratio and the implications for understanding 
particle composition in case I and case II waters," Journal of Geophysical Research: Oceans 106, 14129-14142 (2001).

19. A. Bricaud and A. Morel, "Light attenuation and scattering by phytoplanktonic cells: a theoretical modeling," Applied Optics 25, 571 (1986).

20. D. Stramski and C. D. Mobley, "Effects of microbial particles on oceanic optics: A database of single-particle optical properties," Limnology and Oceanography 42, 538-549 (1997).

21. Y.-H. Ahn, A. Bricaud and A. Morel, "Light backscattering efficiency and related properties of some phytoplankters," Deep Sea Research Part A. Oceanographic Research Papers 39, 1835-1855 (1992).

22. M. Babin, A. Morel, V. Fournier-Sicre, F. Fell and D. Stramski, "Light scattering properties of marine particles in coastal and open ocean waters as related to the particle mass concentration," Limnology and Oceanography 48, 843-859 (2003).

23. R. A. Reynolds, D. Stramski, V. M. Wright and S. B. Woźniak, "Measurements and characterization of particle size distributions in coastal waters," Journal of Geophysical Research 115, (2010).

24. C. E. Junge, Air Chemistry and Radioactivity (Academic Press, 1963).

25. H. Bader, "The hyperbolic distribution of particle sizes," Journal of Geophysical Research 75, 2822-2830 (1970).

26. R. W. Sheldon, A. Prakash and W. H. Sutcliffe Jr., "The size distribution of particles in the ocean," Limnology and Oceanography 17, 327-340 (1972).

27. O. Ulloa, S. Sathyendranath and T. Platt, "Effect of the particle-size distribution on the backscattering ratio in seawater," Applied Optics 33, 7070 (1994).

28. Y. C. Agrawal and H. C. Pottsmith, "Laser diffraction particle sizing in STRESS," Continental Shelf Research 14, 1101-1121 (1994).

29. P. Traykovski, R. Latter and J. D. Irish, "A laboratory evaluation of the laser in situ scattering and transmissometery instrument using natural sediments," Marine Geology 159, 355-367 (1999).

30. Y. C. Agrawal and H. C. Pottsmith, "Instruments for particle size and settling velocity observations in sediment transport," Marine Geology 168, 89-114 (2000).

31. H. C. van de Hulst, Light Scattering By Small Particles (Dover Publications, 2012).

32. C. F. Bohren and D. R. Huffman, Absorption And Scattering Of Light By Small Particles (Wiley, 2013).

33. W. H. Slade, "FASTMIE," http://www.scattport.org/www.scattport.org/index.php/light-scatteringsoftware/mie-type-codes/list/264-fastmie.html. (2006)

34. D. Risović, "Two-component model of sea particle size distribution," Deep Sea Research Part I: Oceanographic Research Papers 40, 1459-1473 (1993).

35. E. Aas, "Refractive index of phytoplankton derived from its metabolite composition," Journal of Plankton Research 18, 2223-2249 (1996)

36. D. Stramski and D. A. Kiefer, "Light scattering by microorganisms in the open ocean," Progress in Oceanography 28, 343-383 (1991).
37. R. E. Green, H. M. Sosik and R. J. Olson, "Contributions of phytoplankton and other particles to inherent optical properties in New England continental shelf waters," Limnology and Oceanography 48, 2377-2391 (2003).

38. M. S. Twardowski, J. M. Sullivan, P. L. Donaghay and J. R. V. Zaneveld, "Microscale Quantification of the Absorption by Dissolved and Particulate Material in Coastal Waters with an ac-9," Journal of Atmospheric and Oceanic Technology 16, 691-707 (1999).

39. J. R. V. Zaneveld, J. C. Kitchen, and C. Moore, "The scattering error correction of reflecting-tube absorption meters," Ocean Optics XII, June 13-15 1994, Bergen, Norway, SPIE, 44-55.

40. R. Röttgers, D. McKee and S. B. Woźniak, "Evaluation of scatter corrections for ac-9 absorption measurements in coastal waters," Methods in Oceanography 7, 21-39 (2013).

41. WET Labs Inc., Absorption and attenuation meter, ac-9, User's Guide (Revision Q) (2008)

42. WET Labs Inc., ac Meter, Protocol Document (Revision Q) (2011)

43. I. Lefering, F. Bengil, C. Trees, R. Röttgers, D. Bowers, A. Nimmo-Smith, J. Schwarz and D. McKee, "Optical closure in marine waters from in situ inherent optical property measurements," Optics Express 24, 14036 (2016).

44. W. S. Pegau, D. Gray and J. R. V. Zaneveld, "Absorption and attenuation of visible and near-infrared light in water: dependence on temperature and salinity," Applied Optics 36, 6035 (1997).

45. I. Lefering, R. Röttgers, C. Utschig, and D. McKee, "Uncertainty budgets for liquid waveguide CDOM absorption measurements," Appl. Opt. 56, 6357-6366 (2017)

46. J. M. Sullivan and M. S. Twardowski, "Angular shape of the oceanic particulate volume scattering function in the backward direction," Applied Optics 48, 6811 (2009).

47. J. M. Sullivan, M. S. Twardowski, J. Ronald, V. Zaneveld, and C. C. Moore, "Measuring optical backscattering in water," in: Light Scattering Reviews 7, edited by: A. A. Kokhanovsky, 189-224 (Springer Berlin Heidelberg, 2013).

48. T. Oishi, "Significant relationship between the backward scattering coefficient of sea water and the scatterance at $120^{\circ}$," Applied Optics 29 , 4658 (1990).

49. E. Boss and W. S. Pegau, "Relationship of light scattering at an angle in the backward direction to the backscattering coefficient," Applied Optics 40, 5503 (2001)

50. WET Labs Inc., Scattering meter, ECO BB9, User's Guide (Revision K) (2010).

51. W. R. Clavano, E. Boss, and L. Karp-Boss, "Inherent optical properties of non-spherical marine-like particles - from theory to observations," in Oceanography and Marine Biology: An Annual Review, R. N. Gibson, R. J. M. Atkinson, and J. D.M. Gordon, eds. (CRC, 2007), Vol. 45, pp. 1-38. 